\title{
Erratum: Closing the window for compressed dark sectors with disappearing charged tracks
}

\author{
Rakhi Mahbubani, ${ }^{a, b}$ Pedro Schwaller ${ }^{c}$ and José Zurita ${ }^{d, e}$ \\ ${ }^{a}$ Theoretical Physics Department, \\ CERN, Geneva, Switzerland \\ ${ }^{b}$ Theoretical Particle Physics Laboratory, Institute of Physics, EPFL, \\ Lausanne, Switzerland \\ ${ }^{c}$ PRISMA Cluster of Excellence $\mathscr{E}$ Mainz Institute for Theoretical Physics, \\ Johannes Gutenberg University, \\ 55099 Mainz, Germany \\ ${ }^{d}$ Institute for Nuclear Physics (IKP), Karlsruhe Institute of Technology, \\ Hermann-von-Helmholtz-Platz 1, D-76344 Eggenstein-Leopoldshafen, Germany \\ ${ }^{e}$ Institute for Theoretical Particle Physics (TTP), Karlsruhe Institute of Technology, \\ Engesserstraße 7, D-76128 Karlsruhe, Germany \\ E-mail: rakhi@cern.ch, pedro.schwaller@uni-mainz.de, \\ jose.zurita@kit.edu
}

ERRATUM TO: JHEP06(2017)119

ARXIV EPRINT: 1703.05327 


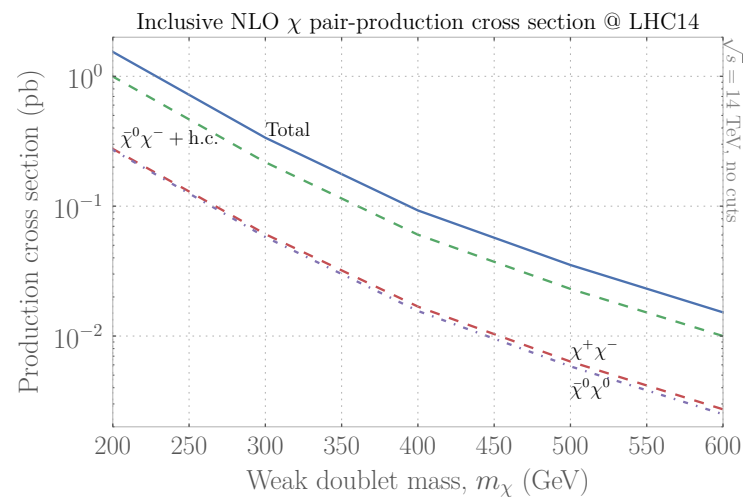

(a)

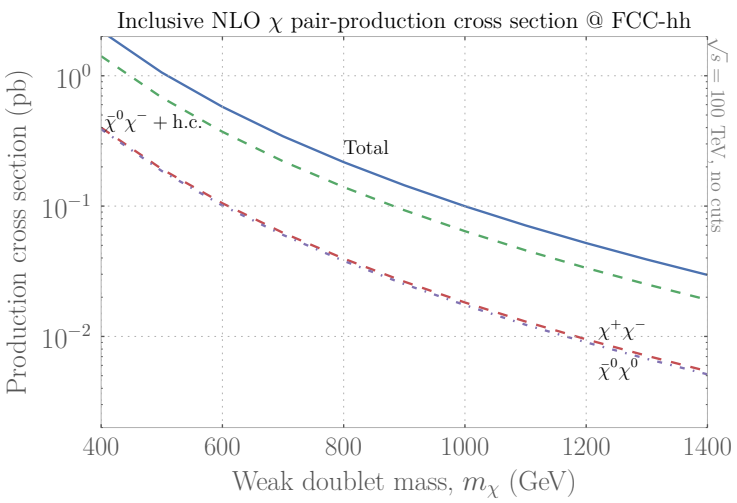

(b)

Figure 1. Corrected pair-production cross sections for a weak doublet with hypercharge $\frac{1}{2}$ at NLO, obtained using PROSPINO in the pure-Higgsino limit, for LHC14 (a) and FCC-hh (b).

The NLO pair-production cross sections for pure higgsinos at LHC14 and FCC-hh shown in figure 2 of our paper were underestimated by $\sim 30 \%$. The corrected cross sections are shown in figure 1 above.

Open Access. This article is distributed under the terms of the Creative Commons Attribution License (CC-BY 4.0), which permits any use, distribution and reproduction in any medium, provided the original author(s) and source are credited. 